\title{
O trabalho como escola: a contribuição preceptoria de terapia ocupacional na residência multiprofissional em saúde da família
}

\author{
The work as a school: the preceptical contribution of \\ occupational therapy in the multiprofessional residence \\ in family health
}

\author{
Renilce Machado dos Santos Araújo', Renato da Costa Teixeira' \\ 'Programa de Pós-graduação Mestrado Profissional em Ensino em Saúde na Amazônia, Universidade do \\ Estado do Pará, Belém (PA) Brasil.
}

\section{RESUMO}

Contextualização: A preceptoria em programas de residência multiprofissional tem representado uma fonte de dados úteis para reflexão e aprimoramento dessa importante prática de integração entre ensino e serviços de saúde. O presente artigo objetiva descrever a experiência da preceptoria com os residentes de Terapia Ocupacional de um programa de residência multiprofissional em saúde da família tendo como cenário um Centro de Saúde-Escola. Descrição da experiência: As ações da preceptoria ocorreram no serviço de Vigilância do desenvolvimento infantil, voltado a prevenção, promoção e detecção precoce de atrasos em crianças de 0 a 12 anos. As atividades iniciaram, com o acolhimento, observação sistematizada da prática e intervenção supervisionada. Os residentes participaram da elaboração de ações de educação em saúde e ações intersetoriais, além de participação na construção de planos de cuidado e estabelecimento de redes de assistência. Foram utilizadas como principais estratégias de ensino, o estudo de caso, a análise da prática profissional e o portfólio reflexivo. Resultados e impactos: A experiência representou uma troca de saberes entre preceptora e residentes sendo possível elaborar e dirigir situações de aprendizagem ajustadas as necessidades de aprendizagem, elaboradas a partir das expectativas e representações dos residentes para favorecimento da relação teoria e prática. Evidenciou-se também que os residentes se mantiveram motivados e assíduos durante a prática no centro-escola, sendo suscitados à problematização das ações de cuidado com os usuários e ainda ao desenvolvimento de estudos referentes à prática, evitando-se oferecer aos mesmos, respostas prontas aos questionamentos. Considerações finais: A atuação da preceptoria em terapia ocupacional foi uma experiência exitosa, a medida em que auxiliou no desenvolvimento das competências essenciais ao exercício da prática do terapeuta ocupacional, bem como ofereceu suporte ao treinamento em serviço, ratificando a necessidade de investimento na atividade de preceptoria e reforçando a relação ensino e serviço no cenário em questão.

PALAVRAS-CHAVE: Preceptoria. Ensino. Atenção primária à saúde. Terapia ocupacional.

\section{RenRESPONDÊNCIA}

Renilce Machado dos Santos Araújo Centro de Saúde Escola do Marco, Universidade do Estado do Pará Av. Rômulo Maiorana, 2558, Marco, CEP 66095-050, Belém (PA), Brasil reni42001@yahoo.com.br

\section{FONTE DE FINANCIAMENTO}

Nenhuma.

\section{CONFLITO DE INTERESSE}

Os autores declararam não

haver conflitos de interesse.

O estudo foi realizado no Centro de Saúde Escola do Marco, Centro de Saúde Escola do Marco,
de Ciências Biológicas e da Saúde, Universidade do Estado do Pará, Belém (PA), Brasil.

\section{ABSTRACT}

Contextualization: The preceptory in multiprofessional residency programs has been a useful data source for reflection and improvement of this important practice of integration between teaching and health services. The present article aims to describe the experience of the preceptory with residents of Occupational Therapy of a multiprofessional family health residency program based on a School Health Center. Description of the experience: The actions of the preceptory occurred in the Child Development Surveillance service, aimed at prevention, promotion and early detection of delays in children from 0 to 12 years. The activities began, with the reception, systematized observation of the practice and supervised intervention. Residents participated in the elaboration of health education actions and intersectoral actions, as well as participation in the construction of care plans and establishment of assistance networks. The main teaching strategies were the case study, the analysis of the professional practice and the reflective portfolio. Results and impacts: The experience represented an exchange of knowledges between preceptor and residents being possible to elaborate and direct learning situations adjusted to the learning needs, elaborated from the expectations and representations of the residents to favor the relation theory and practice. It was also evidenced that the residents remained motivated and assiduous during the practice in the center, being raised to the problematization of the actions of care 
with the users and also to the development of studies referring to the practice, avoiding to offer them, ready answers to the questionings. Final considerations: The performance of the preceptor in occupational therapy was a successful experience, as it assisted in the development of the essential competences to the practice of the occupational therapist, as well as offered in-service training support, ratifying the need for investment in the preceptory activity and reinforcing the teaching and service relationship in the scenario in question.

KEYWORDS: Preceptorship. Education. Primary health care. Occupational therapy.

\section{Contextualização}

Na busca por um perfil profissional ajustado aos princípios do Sistema Único de Saúde (SUS), o Ministério da Saúde e o Ministério da Educação têm elaborado em parceria, diversas políticas com propostas que oportunizam as vivências práticas em ambientes de trabalho. Nesse contexto, destaca-se a figura do preceptor, profissional pertencente ao serviço de saúde que orienta as atividades de graduandos e/ou recém-graduados no momento da prática a partir de objetivo e período definidos ${ }^{1-3}$.

Durante o ensino na prática, o preceptor situa o aluno no seu processo de trabalho e também reflete sobre seu próprio desempenho, porém, é necessário que o mesmo possua clareza do papel que deve desempenhar. Dessa forma, o preceptor precisa ser um facilitador da aprendizagem dos discentes em cenários de prática dispondo de competência para interligar o ensino e o serviço possibilitando aos mesmos a formação de competências para a atuação profissional ${ }^{4,5}$.

Além disso, a preceptoria possibilita a construção de um corpo de conhecimento importante para o crescimento pessoal e profissional tanto para o residente quanto para o preceptor, contribuindo para uma formação e assistência fortalecidas ${ }^{6}$.

No contexto da Atenção Primária à Saúde (APS), os profissionais intervêm de forma estratégica para prevenir doenças e promover a saúde considerando a complexidade do aspecto sócio cultural do usuário, indo muito além do que apenas diagnosticar e tratar patologias. Constituem-se, então, em desafios atuais, a formação de profissionais de saúde nessa perspectiva e também, o treinamento dos profissionais que recebem os discentes nos serviços, para, assim, contribuir para a formação almejada ${ }^{7}$.

A partir da lei 11.129 de 30 de junho de 2005 foram criadas as residências uniprofissionais e a Comissão Nacional de Residência Multiprofissional em Saúde, essa última com o objetivo de regulamentar os programas de residência multiprofissional, que foram instituídos em novembro de 2005 através da portaria interministerial n. $2117^{8,9}$.

As Residências Multiprofissionais em Saúde (RMS) são modalidade de pós-graduação lato sensu que despontam como uma importante estratégia de ensino e que dependem da atuação da preceptoria. Baseados no trabalho em equipe e enfoque interdisciplinar, os programas objetivam proporcionar aos profissionais de saúde atributos que favoreçam o pleno exercício profissional, garantindo o cuidado integral à saúde, a assistência às pessoas e comunidades, a organização da gestão no trabalho e na educação em saúde. Contemplam treze categorias profissionais, incluindo a Terapia Ocupacional ${ }^{7}$.

Mais especificamente, os programas de residência multiprofissional com área de concentração em saúde da família, no qual o terapeuta ocupacional também está integrado, oportunizam a formação voltada à saúde da comunidade a partir de uma inserção social mais ampliada e intervenções baseadas em fatores culturais, econômicos e ambientais. No estado do Pará, a categoria profissional de Terapia Ocupacional atua na residência multiprofissional em saúde da família desde 2012 ${ }^{10,11}$.

Além disso, A preceptoria em programas de residência multiprofissional em cenários de APS tem representado uma fonte de dados úteis para reflexão e aprimoramento dessa importante prática de integração entre ensino e serviços de saúde, bem como a resignificação dos processos de ensino-aprendizagem, contribuindo para uma análise 
mais profunda da especificidade de cada categoria profissional envolvida mesmo diante de um enfoque interdisciplinar ${ }^{4,12}$.

Deter-se-á, pois, na categoria profissional de Terapia Ocupacional, que é uma profissão da área da saúde que auxilia indivíduos, grupos e/ou comunidades a obterem autonomia no desempenho em diversas áreas através da atividade humana ${ }^{13}$ e está em conformidade com os princípios e diretrizes da Atenção Primária no SUS, sobretudo por intervir de maneira direta na prevenção de doenças e agravos e na promoção da saúde dos usuários, conhecendo o contexto de vida dos indivíduos e atuando na capacitação do usuário para que este assuma o papel de có-gestor de seu processo de cuidado ${ }^{14}$.

A fim de que os aprendizes desenvolvam segurança na atuação profissional o preceptor de Terapia Ocupacional, sob a proposta da RMS, estreita a relação teoria e prática, relação em constante transformação, sobretudo no que concerne o objeto de estudo dessa profissão, a atividade humana aos princípios da $\mathrm{APS}^{7,15}$.

Diante do exposto e da relevância da atuação do preceptor em estratégias de ensino em serviço, o presente artigo objetiva descrever a experiência de preceptoria com os residentes de Terapia Ocupacional de um programa de residência multiprofissional em Saúde da Família tendo como cenário um Centro de Saúde-Escola.

\section{Descrição da experiência}

O relato de experiência compreende o período de 2014 a 2017 em um centro de saúde-escola no Munícipio de Belém (PA), o qual é ligado a uma Universidade pública do Pará. O referido centro representa um dos cenários que servem de apoio ao Programa de Residência Multiprofissional em Saúde da Família e apresenta serviços de atenção primária à saúde onde há atuação de equipe multiprofissional, destacando-se a atuação do terapeuta ocupacional.

A ação da preceptoria se concentrou no serviço de Vigilância do Desenvolvimento Infantil, voltado à promoção da saúde, prevenção e detecção precoce de atrasos na infância, sendo realizado o acompanhamento do desenvolvimento global de crianças na faixa etária de 0 a 12 anos, matriculadas no centro-escola ou oriundas de demanda espontânea.

O início da prática dos residentes foi marcado por uma visita institucional, para conhecer as instalações e funcionamento do centro-escola, bem como a apresentação ao gestor e aos demais membros da equipe.

Foram sugeridos pela preceptora e pelos residentes alguns temas para debate relativos aos princípios do SUS, níveis de atenção à saúde, missão institucional do centro de saúde-escola, Terapia Ocupacional na Atenção Primária à Saúde, o processo de avaliação do desenvolvimento neuropsicomotor, fatores de risco para o desenvolvimento infantil e conduta ética na abordagem profissional foram debatidos e precederam às observações sistematizadas e os atendimentos ao público pelos residentes.

Os residentes de Terapia Ocupacional atuavam no serviço por um breve período de três meses, ocorrendo então a permuta. Já na primeira semana, passavam a realizar atividades de agendamento dos atendimentos, evolução em prontuários, realização de anamnese, avaliação e acompanhamento para detecção precoce de alterações dos aspectos motor, cognitivo, interação social, autonomia e desempenho ocupacional nas atividades de vida diária e prática das crianças.

As estratégias de intervenção foram a escuta, o estabelecimento de vínculo e a responsabilização dos cuidadores no processo de cuidado da criança, com prevalência de atendimento individual. Os principais recursos utilizados foram: brinquedos, jogos lúdicos, massa de modelar, papel, lápis de cor e tinta guache, etc. 
Os residentes participaram da elaboração de projetos terapêuticos singulares, estabelecimento de redes de assistência e encaminhamentos das demandas para serviços de média e alta complexidade, além de busca ativa.

Mediante as demandas identificadas, os residentes puderam participar da elaboração de ações de educação em saúde com orientações às famílias a respeito de diversas temáticas como: favorecimento do diálogo tônico e o vínculo entre criança e cuidador; experiências sensório motoras por meio do brincar; construção de recursos para estimulação do aspecto cognitivo, atenção e concentração; cuidados com a rotina de sono; processo de desmame, estimulação da independência nas atividades fisiológicas, prevenção de acidentes domésticos, prevenção de dificuldades escolares e promoção da saúde mental da criança.

Além disso, houve a participação dos residentes em ações de intersetorialidade com visitas às escolas adjacentes ao centro-escola para realizar ações de avaliação e consultoria colaborativa a crianças com dificuldades escolares.

As principais estratégias de ensino utilizadas pela preceptora foram o estudo de caso, a análise da prática profissional e o portfólio reflexivo.

Os estudos de caso correspondiam a uma investigação empírica que permitia o estudo aprofundado das situações vivenciadas em seu contexto real, eram realizados no final de cada semana, sendo selecionados pelos residentes.

Ao final de cada dia, os residentes eram incentivados a refletir sobre a prática, destacando pontos positivos, dúvidas e dificuldades a respeito das atividades, o que gerava pontos de discussão, sugestões para melhoria da prática e ideias de temas para aprofundamento teórico.

A estratégia de portfólio reflexivo consistiu na organização de toda produção resultante dos diversos momentos de aprendizagem e também da reflexão a respeito do desempenho no cenário de prática.

A avaliação dos residentes era processual, com utilização de instrumento padrão para registro de nota, a qual era baseada no desempenho e participação em todas as atividades, sendo reservado um espaço para avaliação da preceptoria pelos próprios residentes.

\section{Resultados e impactos}

A experiência da preceptoria em terapia ocupacional foi exitosa e representou um valioso aprendizado para a preceptora, mesmo com alguns percalços relativos à dificuldade de conciliar as atividades de preceptoria com a assistência aos usuários.

O contato com a residência também motivou a preceptora a buscar mais conhecimentos que pudessem favorecer o aprendizado em serviço e permitiu a correlação com algumas competências de ensino estudadas por Perrenoud, dentre as quais destacam-se: organizar e dirigir situações de aprendizagem, administrar a progressão das aprendizagens, conceber e fazer evoluir dispositivos de diferenciação, competência para saber envolver os alunos em suas aprendizagens e em seu trabalho e competência para trabalhar junto com equipes ${ }^{16}$.

Considera-se que o desenvolvimento de competências e habilidades para o desempenho das ações referentes ao ensino, não é completada na graduação, deve, pois, se estender ao longo de toda a vida profissional de forma contínua e permanente favorecendo uma ação profissional ajustada ao contexto e as necessidades da população ${ }^{17}$.

Dessa forma, os preceptores devem ser foco de investimentos pelas instituições de saúde, sendo importante considerar a motivação dos mesmos para o ensino em serviço e os conhecimentos a respeito de didática e metodologias ativas de aprendizagem com o intuito de orientar melhor o residente durante a prática ${ }^{6}$. 
Nessa perspectiva, é esperada uma troca de saberes e experiências entre o preceptor e o aluno gerando reflexos sobre a prática e a (re)construção do conhecimento ${ }^{18}$.

As atividades de preceptoria oportunizaram aos residentes estabelecer a relação de conteúdos teóricos com a prática vivenciada, sendo que o planejamento das atividades foi construído de acordo com as necessidades de aprendizagem percebidas a partir das expectativas e representações da preceptora e dos residentes.

Essa perspectiva converge para a ideia de que no contexto da Residência Multiprofissional e Saúde (RMS), a figura do preceptor é representada por um sujeito ativo no processo-ensino aprendizagem, favorecendo a interrelação entre os conteúdos teóricos e o mundo do trabalho e aquisição de competências relacionadas à formação profissional ${ }^{18}$.

Os residentes puderam socializar as ações em saúde com a equipe multidisciplinar reforçando o caráter interdisciplinar da atuação dos serviços de APS. Tal fato reflete que prática de saúde nesse nível de atenção à saúde é necessária para ampliar o entendimento de que o cuidado em saúde provém principalmente da correlação com os outros níveis de atenção e não de condutas assistenciais isoladas, sendo oportuno, portanto, perceber como os processos de ensino aprendizagem em APS, tem se configurado e quais meios podem ser pensados para a sistematização e o fortalecimento da preceptoria nos serviços de saúde ${ }^{7}$.

As ações intersetoriais na escola tiveram grande impacto para os residentes que puderam refererir como foi proveitoso se apropriar de novos saberes enfatizando que a abordagem de consultoria da colaborativa como configuração da interrelação entre as áreas de saúde e educação, se faz necessária na eficácia das ações de cuidado ao usário ${ }^{19}$.

Evitou-se a repetição mecânica de procedimentos, priorizando-se um formato de prática diversificado, sendo levados em consideração os aspectos subjetivos de cada residente e valorizando os conhecimentos prévios de cada residente.

Em estudo realizado sobre a preceptoria são referidos dois modelos de ensino que podem ser utilizados pelo preceptor: um tradicional, onde o ensino está centrado no professor preceptor e na simples transmissão de conhecimento e outro modelo chamado emergente no qual é enfatizada a coprodução de autonomia, ao se exercer o cuidado ao usuário, e no desenvolvimento de competências para exercer a vida profissional, com suas respectivas habilidades, demandas e atitudes ${ }^{20}$.

Vale destacar que também foi necessário instaurar regras de convivência, além de dispor de segurança para tecer críticas construtivas, explicitando aos residentes a importância da comunicação clara e objetiva tanto no serviço de Terapia Ocupacional e demais ambientes do centro-escola.

De igual maneira, a experiência da preceptoria demonstrou que é necessário a ação de flexibilizar o planejamento para os residentes convergindo para a ideia de nessa atividade de ensino não há transferência de conhecimentos e sim um momento para se construir novos conhecimentos ${ }^{21}$.

A experiência da preceptoria no centro-escola se aproximou das ideias de Carlos Brandão a respeito do processo de educação ao considerar que os homens estão sempre aprendendo uns com os outros ${ }^{22}$, pois na preceptoria tanto o residente quanto o preceptor têm participação ativa e complementar no processo de ensino aprendizagem, o que foi percebido e amplamente referido pelos sujeitos envolvidos.

Mesmo assim, as dificuldades apenas impulsionaram o ensejo de preservar o serviço de Terapia Ocupacional do centro-escola como espaço propício ao aprendizado significativo dos residentes, colaborando para a construção de um perfil de competências para a preceptoria no contexto dessa profissão no âmbito da APS. 


\section{Considerações finais}

O presente relato indicou que a atuação da preceptoria de Terapia Ocupacional no contexto da residência multiprofissional em Saúde da Família foi uma experiência exitosa de ensino em serviço para os residentes. A preceptoria ofereceu suporte ao treinamento em serviço para os residentes, auxiliando-os no acolhimento de demandas, no estabelecimento da relação terapeuta-usuário, na atuação em equipe e na construção de redes de assistência em saúde; características enfatizadas na Política Nacional de Atenção Básica; bem como, contribuiu para a elaboração de planos de cuidado ajustados ao contexto de vida da população atendida ações de educação em saúde com orientações às famílias sobre como estimular os aspectos do desenvolvimento infantil; participaram da elaboração de projetos terapêuticos singulares e ainda do estabelecimento de redes de assistência para resolução de demandas e ações intersetorial, e ainda, bem como possibilitou uma importante fonte reflexão e aprimoramento de sua própria intervenção no serviço em questão, reforçando a relação ensino e serviço no centro-escola e finalmente ratificando a necessidade de mais investimentos em propostas de educação permanente para preceptores.

\section{Referências}

1. Aguiar AC, Borges WC, Araujo EFS, Braga EB, Kalil IL. Introdução à pesquisa "preceptoria em programas de residência no Brasil e na Espanha: ensino, pesquisa e gestão" premissas, opções metodológicas e caracterização dos respondentes. In: Aguiar AC, organization. Preceptoria em Programas de residência: ensino, pesquisa e gestão. Rio de janeiro: CEPESC/ IMS/UFRJ; 2017. Capítulo 1. 207 p.

2. Souza MCA, Costa EMA, Mendonça MA, Gonçalves SJC, Júnior JCSC, Silva MAM. Inovação metodológica na integração ensino-serviço na educação médica. RMRP. 2016;49(3):284-91. http://dx.doi.org/10.11606/issn.2176-7262.v49i3p284-291.

3. Botti SHO, Rego S. Preceptor, supervisor, tutor e mentor: quais são seus papéis? Rev Bras de Educ Méd. 2008;32(3): 363-73.

4. Ferreira FC, Dantas FC, Valente GSC. Saberes e competências do enfermeiro para preceptoria em unidade básica de saúde. Ver Bras Enferm. 2018;71(4):1657-65.

5. Teixeira RC, Souza RJ, Kietzer KS, Araújo RMS, Gonçalves KLP. Preceptoria em Saúde: definição do papel do preceptor. Anais do XXVIII Fórum nacional de ensino em Fisioterapia. V Congresso brasileiro de educação em Fisioterapia. VII Congresso nacional de Fisioterapia na saúde coletiva. Supl Cader Educ Sau Fisio Ed Rede Unida. 2018;5(10):71-72.

6. Silva RM, Freitas LS, Araújo CLS, et al. Importância da residência de enfermagem no processo ensino-aprendizagem: uma revisão integrativa. Rev Enf At. 2018;2018:86.

7. Izecksohn MMV, Teixeira JE Jr, Stelet BP, Jantsch AG. Preceptoria em medicina de família e comunidade: desafios e realizações de uma atenção primária à saúde em construção. Ciên Saúd Colet. 2017;22(3):737-46. http://dx.doi. org/10.1590/1413-81232017223.332372016.

8. Brasil. Presidência da República. Casa Civil. Subchefia para Assuntos Jurídicos. Lei n. 11.129, de 30 de junho de 2005 . Institui o Programa Nacional de Inclusão de Jovens-Pró-Jovem: cria o Conselho Nacional de Juventude e altera as leis 10.683, de 28 de maio de 2003 e 10.429, de 24 de abril de 2002 e dá outras providências. Diário Oficial da União [Internet]. Brasília, 30 jun. 2005 [citado em 15 Abr de 2019]. Disponível em: http://www.planalto.gov.br/ccvil_03/_ato2004-2006/2005/lei11129. htm.

9. Brasil. Portaria Interministerial n. 2117/05, de 03 de novembro de 2005. Institui no âmbito do Ministério da Saúde e Ministério da Educação a residência multiprofissional em saúde. Diário Oficial da União, Brasília, DF, 04 nov. 2005. Poder Executivo.

10. Silva AGI, Santos MIPO. Projeto Político Pedagógico do Programa de Residência Multiprofissional em Saúde da Família da Universidade do Estado do Pará. Belém. 2015.

11. Brasil. Mistério da Saúde. Secretaria de Gestão do Trabalho e da Educação na Saúde. Departamento de gestão da Educação na Saúde. Residência Multiprofissional em saúde: experiências, avanços e desafios. Brasília: Ministério da Saúde; 2006. $414 \mathrm{p}$.

12. Paiva LFA, Souza FR, Savioli KC, Vieira JL. A Terapia Ocupacional em saúde da família e comunidade. Cad Ter Ocupa UFSCAR. 2013;21(3):595-600. http://dx.doi.org/10.4322/cto.2013.061.

13. Della Barba PCS, Silva RF, Joaquim RHVT, Brito CMD. Formação inovadora em Terapia Ocupacional. Interf Comunic Saú Educ. 2012;16(42):829-42.

14. Jardim TA, Afonso VC, Pires IC. A Terapia Ocupacional na estratégia saúde da família-evidências de um estudo de caso no Município de São Paulo. Ver Ter Ocup Univ São Paulo. 2008;19(3):167-75.

15. Onório JLS, Bezerra WC, Santos EMS, Silva EM. O exercício da preceptoria na formação do terapeuta ocupacional. Rev Bras Ens Sup. 2017;3(4):79-96. http://dx.doi.org/10.18256/2447-3944.2017.v3i4.2131.

16. Perrenoud P. Dez novas competências para ensinar - convite à viagem. Porto Alegre (RS): Artmed; 2000. 192 p. (Tradução Patrícia Chittoni Ramos).

17. Valente GSC, Viana LO. O ensino de nível superior no Brasil e as competências docentes: um olhar reflexivo sobre essa prática. Prax Educ. 2010;6(9):209-26. 


\section{ighe}

18. Ribeiro KRB, Prado MI. A prática educativa dos preceptores nas residências em saúde: um estudo de reflexão. Rev Gaúcha Enferm. 2013;34(4):161-5. PMid:25080714.

19. Folha DRSC, Monteiro GS. Terapia Ocupacional na atenção primária à saúde do escolar visando a inclusão escolar de crianças com dificuldades de aprendizagem. Ver Interinst Bras Ter Ocup Riso. 2017;1(2):202-20.

20. Barreto VHL, Monteiro ROS, Magalhães GSG, Almeida RCC, Souza LN. Papel do preceptor da atenção primária em saúde na formação da graduação e pós-graduação da universidade federal de Pernanbuco. Pernambuco: um termo de referência. Rev Bras Educ Med. 2011;35(4):578-83. http://dx.doi.org/10.1590/S0100-55022011000400019.

21. Freire P. Pedagogia da Autonomia: saberes necessários à prática educativa. São Paulo. Ed Paz e Terra; 1996.

22. Silva AA, Souza KR. Educação, pesquisa participante e saúde: as ideias de Carlos Rodrigues Brandão. Trab Educ Saúd Rio de Janeiro. 2014;12(3):519-539.

\section{Contribuição dos autores}

Renilce Machado dos Santos Araújo participou da concepção da pesquisa, da elaboração do desenho metodológico, do desenvolvimento da pesquisa, bem como da elaboração do manustrito. Dr Renato da Costa Teixeira foi responsável pela supervisão da pesquisa e revisão crítica do manuscrito. 